\title{
Routing Protocols for Wireless Sensor Networks
}

\author{
Chaitanya Mankar ${ }^{1}$, Vidhya Dhamdhere ${ }^{2}$ \\ ${ }^{1}$ MECN, G. H. Raisoni College of Engineering and Management (GHRCEM), India \\ ${ }^{2}$ Faculty, Computer Department, G.H.Raisoni College of Engineering and Management (GHRCEM), India
}

\begin{abstract}
The development of wireless sensor networks (WSN) was originally motivated by military applications such as battlefield surveillance. However, wireless sensor networks are now used in many civilian application areas, including environment and habitat monitoring, healthcare applications, home automation, and traffic control. In last few years there are lots of advance research has done in WSNs. But the most of research is emphasis on how WSN achieve high efficiency with minimal energy use for this there are number of routing protocol are developed in WNSs depending upon the application and architecture of the network system. In this review article, we discuss the wireless sensor node architecture, WSNs Protocol stack. Further we discuss Routing challenges and design issues in WSN and last we categorize the routing protocols into three main categories such as protocol operation, network organization and route discovery
\end{abstract}

Keywords: Flooding, Gossiping, Gear, Leach, Pegasis, Wireless Sensor Networks

\section{Introduction}

Sensing is technique to gather information about physical object or area. Sensor (transducer) is object performing a sensing task, converting one form of energy into other from. Human body consist number of natural sensor such as Eyes, Ears, Nose, skin. A wireless sensor network consists of hundreds or thousands of low cost sensor nodes which could either have a fixed location or randomly deployed to monitor the environment. A sensor network is an organization consists of sensing technique, computing technique, and communication elements that give an ability to inspect, watch, and react. There are four basic components in a sensor network: (1) sensors (2) communication network (3) Base station or Sink (4) a set of computing resources at the central point to handle data correlation, event trending, status querying, and data mining

\subsection{Sensor Node Architecture}

A basic sensor node comprises five main components Controller A controller to process all the relevant data, capable of executing arbitrary code. Memory Is required for

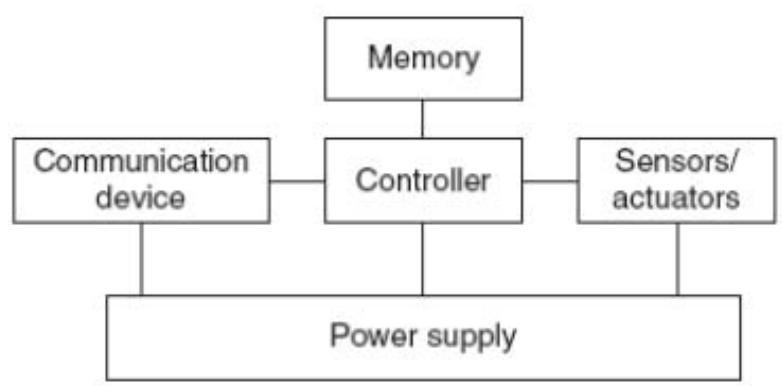

Figure 1: Sensor Node Architecture

storing information and programs usually, different types of memory are used for programs and data. Sensors and actuators Is devices that can observe or control physical parameters of the environment. Communication Device For shearing information over wireless network communication device is used. Power supply some forms of batteries are necessary to provide energy. Sometimes, some form of recharging by obtaining energy from the environment is available as well (e.g. solar cells).

\subsection{Architecture of the protocol stack for wireless sensor network}

The architecture of protocol stack used by the sink and sensor nodes is shown in Fig 2. sink is also called as base station (bs). A base station links the sensor network to another network (like a gateway) to disseminate the data sensed for further processing. Base stations have enhanced capabilities over simple sensor nodes since they must do complex data processing. This protocol stack (Fig.2) is made up of physical layer, data link layer, network layer, transport layer, application layer. The physical layer addresses the needs of a robust modulation, transmission and receiving techniques. Responsibilities of MAC layer include decide when a node accesses a shared medium, resolve any potential conflicts between competing nodes, correct communication errors occurring at the physical layer, Responsibility of Network layer include Routing is a key responsibility of the network layer Routing protocol is responsible for finding and maintaining path from sensor to sink In network layer there are two communication model present as below 1)Direct communication model: every sensors communicates directly with the sink device often not feasible due to lack of energy, scale of network, lack of unobstructed communication links between sensors and sink 2)Multi-hop communication model: sensors cooperate in propagating sensor data towards the sink perform other activities such as framing, addressing, and flow control supplied by the transport layer. The transport layer helps to maintain the flow of data if the wireless sensor network application requires it. Depending on the sensing tasks, different types of application Software can be set up and used on the application layer [4] 


\begin{tabular}{|c|}
\hline Application Layer \\
\hline Transport Layer \\
\hline Network Layer \\
\hline Data link Layer(MAC Layer) \\
\hline Physical Layer \\
\hline
\end{tabular}

Figure 2: WSN Protocol stack

\section{Routing Challenges and Design Issues}

Depending on the application, different architectures and design constraints have been considered for sensor networks. Since the performance of a routing protocol is closely related to the architectural model, in this section we study design issues in sensor networks.

\subsection{Node Deployment}

Sensor node deployment in WSNs is application dependent and can be either manual or random which finally affects the performance of the routing protocol. In most applications, sensor nodes can be scattered randomly. If the resultant distribution of nodes is not uniform, optimal clustering becomes necessary to allow connectivity and enable energy efficient network operation.

\subsection{Energy Consumption}

Since the transmission power of a wireless radio is proportional to distance squared or even higher order in the presence of obstacles, multi-hop routing will consume less energy than direct communication However; multi-hop routing introduces significant overhead for topology management and medium access control. Direct routing would perform well enough if all the nodes were very close to the sink. Most of the time sensors are scattered randomly over an area of interest and multi-hop routing becomes unavoidable.

\subsection{Scalability}

Routing protocols should be able to scale with the network size. Also, sensors may not necessarily have the same capabilities in terms of energy, processing, sensing, and particularly communication. Hence, communication links between sensors may not be symmetric, that is, a pair of sensors may not be able to have communication in both directions. This should be taken care of in the routing protocols.

\subsection{Network Dynamics}

In many applications, the movement of sensor nodes or the base station is essential. This means that sensor nodes are moving nodes (i.e., not stationary as assumed by many of network architectures). This has arisen the routing stability issues as well as energy, bandwidth, etc.

\subsection{Transmission Media}

In a multi-hop sensor network, a wireless medium is used to link nodes for communications goal. These links can be formed by radio, Infrared which is license free and robust to interference from electrical devices, and Optical media.

\subsection{Coverage}

The sensor nodes view of the environment that it is situated in is limited both in range and in accuracy. This means the ability of sensor nodes to cover physical area of the environment is limited.

\subsection{Data Aggregation}

If the data classification and fusion can be complete quickly in sensor node, it helps in efficient query processing and decreases network overhead dramatically

\section{Classification of Routing Protocol}

In Wireless sensor Network Routing can be classified in three categories according to Network Structure, Route Establishment and a Protocol operation .Following diagram show the classification of WSNS routing protocol. In general routing in WNS can be dividing into three categories named as Flat-Based, Hierarchical-Based and location-Based[6]. In Flat-Based routing protocol all node have same role. In Hierarchical-Based routing protocol node have different role according to structure of network. in location- Based Routing protocol sensor node position are exposed to route data..Furthermore WSN protocols can be classified into three categories, namely reactive, proactive and hybrid protocols depending on how the source sends a route to the destination. In reactive protocols, routes are computed on demand In routing proactive protocols all routes are computed before they are really needed, while. Hybrid protocols use a combination of these two approaches. Another possibility is to classify the protocols with respect to the protocol operation. Protocol operation can be Negotiation Based, Multipath Based, Query Based, QoS based and Coherent Based 


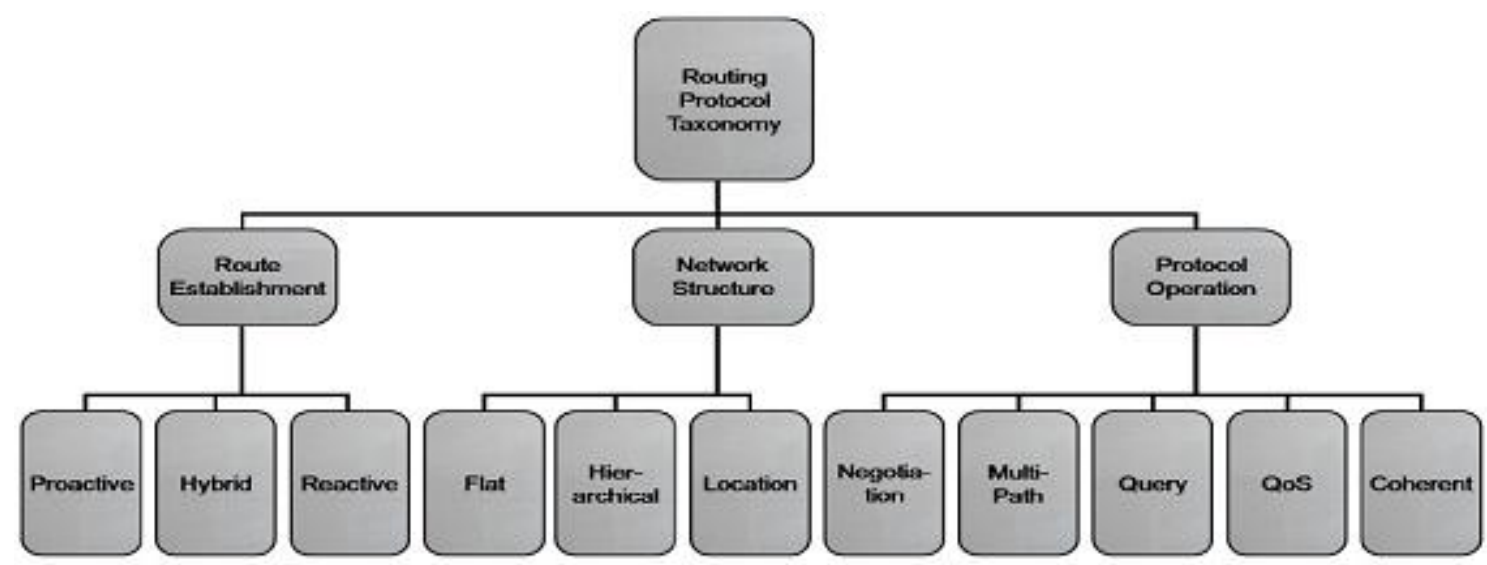

Figure 3: Classification of Routing Protocol

\subsection{Routing Protocol in Wireless Sensor Network}

1) Flooding [2]: Flooding is oldest protocol in wireless sensor network it is simplest routing protocol. It requires each node in the network to broadcast a packet upon receiving it from the first time However there are some deficiencies for routing the packet in network 1) Implosion: in implosion case a duplicated packet are send to same node 2) Overlap: if two sensor node cover an overlapping measuring region, both of them will sense the same data as a result their neighbour node will receive duplicated data 3 ) Resource Blinding: in flooding nodes do not take into account the amount of energy resources available to them at a given time

2) Gossiping [2]: .In gossiping node can forward the incoming data to randomly selected neighbour node. Once the gossiping node receive the message it can forward the data back to the neighbour or to the another randomly selected neighbour node Gossiping can solve the implosion problem and energy conservation problem but it cannot avoid the overlapping problem .There is another problem in gossiping the cost of long time propagation for sending message

3) Direct Diffusion [7]: Direct diffusion is aggregation, datacentric and application aware routing protocol. At the starts of the directed diffusion process, the base station specifies a low data rate for incoming packet. After that, the base station can reinforce one particular sensor to send events with a higher data rate by resending the original interest message with a smaller interval. Direct diffusion consist of four important element as follows 1) Interest: an interest message specifies what user want it consist of timestamp field and gradient fields 2) Data: is the collected or processed information data is named using attribute value pair 3) Gradient:-is direction state created in each node that receive interest message 4) Reinforcement: After the gradient direction is set towards the neighbouring node from which interest is receive. Event start following toward the origin of interest along multiple gradient path the sensor network reinforce small number of path

\section{Advantages}

1) On demand route setup

2) Each node does aggregation and caching, thus
3) Good energy efficiency and low delay

Disadvantages

1) Query-driven, not a good choice for continuous data delivery

2) Extra overhead for data matching and queries

4) SPIN: Sensor Protocol for Information via Negotiation SPIN is based on data centric routing where the sensor node send ADV message when data is available to node and wait for REQ message . SPIN contain three types of message

1)ADV: When node want to send data it advertise via broadcasting this message to all node in the network

2)REQ: If any node interest to receive data then that node send REQ message

3)DATA: DATA message contain actual data along with metadata header

Advantages

1) Solve the classic problems

2) Topological changes are localized

Disadvantages

3) No guarantee on the delivery of data

5) LEACH[2]: Hinzelman introduced a hierarchical clustering algorithm for sensor network called low energy adaptive cluster hierarchy-based protocol .In leach protocol the node can play different role in the network In LEACH the operation is divided into rounds, during each round different set of node are selected as cluster head Once a node selected as a cluster head again for $p$ round at the end of each round each round node which is not cluster head is select the nearest cluster head and transmit the data to that particular cluster head after receiving data from all the non-cluster head then cluster head forward the data to base station

\section{Advantages}

1) Distributed and no global knowledge of network Required

Disadvantages

1) Extra overhead to do dynamic clustering

6) PEGASIS [8]: (Power -Efficient Gathering in sensor information System) PEGASIS is a chain based power efficient protocol inspired from LEACH protocol. In

\section{Volume 5 Issue 1 January 2016}




\section{International Journal of Science and Research (IJSR) \\ ISSN (Online): 2319-7064}

Index Copernicus Value (2013): 6.14 | Impact Factor (2014): 5.611

PEGASIS each node only communicates with a close neighbour. The sensor nodes are homogenous and energy constrained with uniform energy. The base station is fixed at a far distance from the sensor. In PEGASIS each node can take turn of being a leader of chain where chain can be constructed using greedy algorithm. PEGASIS assume that sensor node have global knowledge of network, nodes are stationary and node have location information about all other node

\section{Advantages}

1) Eliminate the overhead of dynamic cluster formation

2) Minimizing the total sum of transmission distances

3) Limiting the number of transmissions

Disadvantages

1) To obtain a global knowledge is difficult

2) It is not suitable for sensor networks

3) Scalability problem

4) Very long delay

7) GEAR [11]: Location based routing protocols for sensor network need location information of all the sensor nodes to calculate the distance between any two nodes. GEAR is a location based routing protocol which uses GIS (Geographical Information System) to find the location of sensor nodes in the network. According to this protocol, each node stores two types of cost of reaching the destination: estimated cost and learning cost. The estimated cost is a combination of residual energy and distance to destination. The learned cost is a modified estimated cost and it accounts the routing around holes in the network. When a node does not have any closure neighbours towards the target region, a hole occurs. In case where no holes exit, the estimated cost is equal to the learned cost. The GEAR protocol only considers a certain region rather than sending the interests to the whole network as happens in Directed Diffusion and thus restricting the number of interests.

8) TEEN (Threshold sensitive energy efficient sensor network protocol)[11]The sensor network architecture is based on a hierarchical grouping where closer nodes from clusters and this process goes on the second level until base station is reached. TEEN is not good for applications where periodic reports are needed since the user may not get any data at all thresholds are not reached. The architecture of APTEEN (Adaptive threshold sensitive energy efficient sensor network protocol)[10] is same as TEEN. APTEEN supports three different query types: historical, to analyze past data values, one time, to take a snapshot view of the network and persistent to monitor an event for a period of time.

9) HEED (Hybrid energy efficient distributed clustering)[12] is a clustering protocol for WSNs, which extends the basic scheme of LEACH by using residual energy as a primary parameter and network topology features (e.g. node degree ,distances to neighbours) as secondary parameter to break tie between candidate cluster heads, as a metric for cluster selection to achieve power balancing. That means the cluster heads are probabilistically selected based on their residual energy and sensor nodes join the clusters according to their power level. The clustering process is divided into lot of iterations and in each iteration; nodes which are not covered by any cluster head double their probability of becoming cluster head. Since this energy efficient clustering protocol enable every node to independently and probabilistically decide on its role in the clustered network, They can't guarantee optimal elected set of cluster heads. The primary goals of HEED are prolonging network life-time by distributing energy consumption, terminating the clustering process within a constant number of iterations/steps, minimizing control overhead, and producing well-distributed cluster heads and compact clusters. HEED distribution of energy extends the lifetime of nodes within the network thus stabilizing the neighboring node. SECA (Saving energy clustering algorithm) [3] is used to provide efficient energy consumption in WSNs. In order to make an ideal distribution for sensor node clusters, authors calculates the average distance between the sensor nodes and take into residual

\section{Conclusion}

In our work, first we have gone through a survey of routing techniques in wireless sensor networks. The routing techniques are classified as proactive, reactive and hybrid based on their Route Establishment. Further, these are classified as Network Structure as Flat-Based, HierarchicalBased and location-Based structure, Protocol operation can be classified on Negotiation Based, Multipath Based, Query Based, QoS based and Coherent Based . In this document we have discussed seven routing protocols these seven protocols are FLODDING, GOSSIPING, DIRECT DIFFUSION, SPIN, LEACH, PEGASIS, GEAR, TEEN, HEED. Since the sensor networks are application specific, we can't say any particular protocol is better than other. We can compare these protocols with respect to some parameters only. The efficient protocol provided the most optimized solution but still holds some major drawbacks as discussed above. So we have seen that though these protocols are fairly efficient but still there's a need to develop a more efficient protocol which removes all the flaws described above.

\section{References}

[1] Holger Karl and Andreas Willig. "Protocols and Architecture for Wireless sensor networks," Wiley, 005.ISBN:0470095105

[2] Kemal Akkaya , Mohamed Younis, "A survey on routing protocols for wireless sensor networks," 2003 Elsevier B.V.

[3] Wendi RabinerHeinzelman, AnanthaChandrakasan, and HariBalakrishnan, "Energy-Efficient Communication Protocol For Wireless Microsensor Networks", Proceedings of the 33rd Hawaii

[4] J.H. Chang, L. Tassiulas, "Maximum lifetime routing wireless sensor networks," in Proceedings of the Advanced Telecommunications and Information Distribution Research, College Park, MD, 2000 International Conference on System Sciences - 2000

[5] Jennifer Yick, Biswanath Mukherjee, Dipak Ghosal, "Wireless sensor network survey," Computer Networks, Elsevier, vol. 52, pp. 2292-2330, 2008. 
[6] W. Heinemann, A. Chandrakasan, H. Balakrishnan, Energy-efficient communication protocol for wireless Sensor networks, in: Proceeding of the Hawaii International Conference System Sciences, Hawaii, January 2000.

[7] D. Braginsky, D. Estrin, "Rumor routing algorithm sensor networks," in Proceedings of the First Workshop on Sensor Networks and Applications (WSNA), Atlanta, 2002

[8] S. Lindesy and C. Raghavendra, "PEGASIS: PowerEfficient Gathering in Sensor Information System," in Proceedings of the Aerospace Conference, IEEE, vol. 3, Big Sky, Montana, 2002

[9] C.Rahul,J. Rabaey, "Energy Aware Routing for Low Energy Ad Hoc Sensor Networks," in Wireless Communications and Networking Conference,IEEE, vol. 1, 2002, pp. 350-355.

[10] Younis, S Fahmy, "HEED: a hybrid, energy-efficient, distributed clustering approach for ad hoc sensor networks", IEEE Trans Mob Comput. 3(4), 366-379 (2004). doi:10.1109/TMC.2004.41

[11] Xuegong, C Yan, "A control algorithm based on double cluster-head for heterogeneous wireless sensor network", Proc Industrial and Information Systems (IIS), pp. 541-544 (July 2010)

[12] F Akyildiz, W Su, Y Sankarasubramaniam, ECayirci, "A survey on sensor networks", IEEE Commun Mag. 40(8), 102-114 (2002). Doi: 10.1109/ MCOM.2002.1024422

[13] au-Yang Chang and Pei-Hao Ju, “An efficient cluster based power saving scheme for wireless sensor networks", EURASIP journal on wireless comm. And net 2012

[14] N Al-Karaki, AE Kamal, "Routing techniques in wireless sensor networks: a survey", IEEE Wirel Commun. 11(6), 6-28 (2004). Doi: 10.1109 /MWC. 2004.1368893

[15] Zhu, H Wang, "An improved K-means clustering algorithm", Proc 2nd IEEE International Conference Information Management and Engineering (ICIME), pp. 190-192 (2010)

\section{Author Profile}

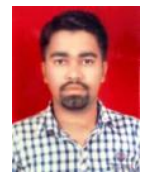

Chaitanya Mankar received the B.E. degree in Computer Engineering Sinhgad Instittute of Technology, Pune. He is currently doing his M.E in Computer Networks in G. H. Raisoni College of engg. \& Mgmt, Pune, (MH). His Research area Wireless Sensor Network, Wireless Network, Network Communication. 